\title{
Is radiation exposure during sacral neuromodulation within safety limits?
}

Ali Alabbad; Elie Abdo; and Magdy Hassouna

University of Toronto, Toronto, ON, Canada

Cite as: Can Urol Assoc J 2018 August 30; Epub ahead of print. http://dx.doi.org/10.5489/cuaj.5262

Published online August 30, 2018

Abstract was previously presented at the 2017 International Continence Society meeting, Sept.12-15, 2017, Florence, Italy.

***

\section{Introduction}

The U.S. Food and Drug Administration (FDA) approved Sacral Neuromodulation (SNM) for intractable urge incontinence in 1997, urgency/ frequency syndrome, and non-obstructive urinary retention in 1999 for Patients who have failed to respond or could not tolerate conservative treatment [1]. In 2011, the FDA approved SNM for chronic fecal incontinence in patients who have failed or could not tolerate conservative treatment.

Fluoroscopy guidance is recommended during permanent tined lead insertion in the S3 foramen. Before InterStim system implantation, the patient underwent a clinical trial either by basic evaluation using temporary lead in the S3 foramen, (also referred to as Peripheral Nerve Evaluation or PNE) which is a simple, in-office procedure for external stimulation or by advanced evaluation using permeant tined lead in the S3 foramen under fluoroscopy guidance (also referred to as Stage I) which is initiated through an outpatient procedure performed in a hospital or surgical center for external stimulation. Both evaluations are short-term, and the effects are reversible by removing the leads or turning off the device. Stage II, is implantation of a subcutaneous implantable pulse generator (IPG). If PNE was performed, fluoroscopic confirmation for the new permanent tined electrode placed in the S3 foramen is advised followed by the implantation of a subcutaneous IPG in the same sitting.Fluoroscopy utilizes X-rays, which are high energy ionizing radiations which causes cellular damage and even cell death. The amount of damage depends upon the total dose, duration of exposure, and the site of exposure. This damage can lead to biological effects, which may be stochastic (independent of the dosage received) or deterministic (dose-dependent effects) [2, 3]. The major source of radiation is the Carm, which is used to produce images for surgical guidance. The radiation exposure can be direct or indirect. Direct exposure is when the person is in the line of the radiation rays produced by the fluoroscopy machine. Indirect exposure occurs from scattered rays resulting from the interaction 
between the primary beam and the patient which disseminate in all directions $[3,4]$. The guidelines approved in 2009 by the Society of Interventional Radiology (SIR) and Cardiovascular \& Interventional Radiology Society of Europe (CIRSE) which identify patients with potential skin injuries requiring clinical follow-up (peak skin dose $>3 \mathrm{~Gy}$, air kerma at the patient entrance reference point $>5 \mathrm{~Gy}$, kerma area product $>500 \mathrm{~Gy} \cdot \mathrm{cm}^{2}$, or fluoroscopy time $>60$ minutes)[5].

\section{Methods}

The medical charts of patients who underwent InterStim implantation performed by one surgeon, his trained fellows, or residents between January 2014 and July 2016 were reviewed retrospectively. Approval was obtained from the Research Ethic Board of the UHN \# 16-5889AE. Patients' demographic data, Body mass index (BMI), indication of treatment, radiation dose data (fluoroscopy time (FT), cumulative dose (CD) which also known as air kerma at the patient entrance reference point (usually measured in mGy), and dose area product (DAP) which also known as kerma area product (usually measured in Gy.cm ${ }^{2}$ )), the nature of the surgery and operation time were collected. The results were compared to radiation exposure during ureteroscopy (FT: 44 sec,DAP: 6.01 Gy.cm ${ }^{2}$,CD: 12 mGy)[6,7,8].

\section{Results}

141 medical charts were reviewed, 83 patients were included in our study, and 58 patients were excluded due to insufficient radiation dose data. The fluoroscopy machine utilized during tined lead insertion was the General electric OEC 9900 Elite. The majority of subjects were females (67.5\%), mean age was 58.3 years (range 21-86 years, SD 14), and mean BMI was 28.9 $\mathrm{kg} / \mathrm{m}^{2}$ (SD 6). The indications of the treatments were as follows: Overactive bladder syndrome (50.6\%), Idiopathic urinary retention (36.2\%), Painful bladder syndrome (7.2\%), Fecal incontinence (4.8\%), and Nocturnal enuresis (1.2\%). Full implantation was the most common surgery (47\%) followed by stage implantation (34.9\%), Revision (17\%), and Twin implantation (1.2\%). The mean operation time was 37.16 minutes (21-69 min, SD 10). The FT was measured in 83 patients: the mean FT was 31.03 seconds (9.5 -155 sec, SD 20). The CD was measured in 50 patients: the mean CD was $13.36 \mathrm{mGy}$ (2.11-33.11 mGy, SD 9). DAP was measured in 33 patients, mean DAP $3.97 \mathrm{~Gy} \cdot \mathrm{cm}^{2}\left(0.5995-24.93 \mathrm{~Gy} \cdot \mathrm{cm}^{2}\right.$, SD 4). So radiation exposure during InterStim implantation is comparable to radiation exposure during ureteroscopy and based on the guidelines approved in 2009 by the SIR and CIRSE which identify patients with potential skin injuries requiring clinical follow-up; the FT, CD and DAP during interStim implantation were well within the threshold level for radiation exposure (peak skin dose > $3 \mathrm{~Gy}$, air kerma at the patient entrance reference point $>5 \mathrm{~Gy}$, kerma area product $>500 \mathrm{~Gy} \cdot \mathrm{cm}^{2}$, or fluoroscopy time $>60$ minutes).

\section{Discussion}


The International Commission on Radiation Protection (ICRP) recommends guidelines for radiation exposure. For medical personnel, it has been recommended that the exposure should not exceed $20 \mathrm{mSv}$ per year. The maximum duration for which this level of exposure is allowed is 5 years, hence a maximal total body exposure over 5 years should not exceed $100 \mathrm{mSv}$. "Radiation exposure is minimal during sacral neuromodulation, the cumulative dose for physicians should not be ignored. With a mean exposure of $1.7 \mathrm{mrem}$ during an initial lead placement, a physician is exposed to the equivalent of one chest x-ray with every 4.7 procedures" [9].

We are aware of the limitations in our study as it is a retrospective study with a small sample size.

\section{Conclusion}

The level of radiation exposure patients encounter during InterStim implantation is minimal and comparable to the level of radiation exposure patients encounter during ureteroscopy. 


\section{References}

1. Shaker HS, Hassouna M. Sacral root neuromodulation in idiopathic nonobstructive chronic urinary retention. J Urol. 1998; 159(5):1476-8.

2. Jindal, T. (2013). The risk of radiation exposure to assisting staff in urological procedures:A literature review. Urologic Nursing, 33(3), 136-139, 147.

3. Rehani, M.M., Ciraj-Bjelac, O., Vañó, E.,Miller, D.L., Walsh, S., Giordano,B.D., \& Persliden, J. (2010). Radiol -ogical protection in fluoroscopically guided procedures performed outside the imaging department.Annals of the ICRP, 40(6), 1-102.

4. Kumari, G., Kumar, P., Wadhwa, P., Aron,M., Gupta, N.P., \& Dogra, P.N. (2006).Radiation exposure to the patient and operating room personnel during percutaneous nephrolithotomy.Inernational Urology and Nephrol -ogy, 38(2),207-210.

5. Vano E, Fernandez JM, Sanchez RM, Martinez D, lbor LL, Gil A, Serna-Candel C .Patient radiation dose management in the follow-up of potential skin injuries in neuroradiology. AJNR Am J Neuroradiol.2013 Feb; 34(2):277-82.

6. Hadjipavlou, Marioslam, Vincent et al. Predicting radiation exposure in ureterorenoscopy and laser lithotripsy :An analysis of patient and stone characteristics .The Journal of Urology 2015, Volume 193, Issue 4, e317.

7. Pricop C, Maier A, Negru D, et al. Extracorporeal shock waves lithotripsy versus retrograde ureteroscopy: is radiation exposure a criterion when we choose which modern treatment to apply for ureteric stones? Bosnian Journal of Basic Medical Sciences. 2014;14(4):254-258.

8. J. Hristova-Popova , I. Saltirov, K. Petkova ,J. Vassileva. Radiation exposure to staff and patients during two endourological procedures. European Medical Physics and Engineering Conference, Sofia 2012.

9. Alana Murphy, Courtney Lee, Kevin Wunderle Howard Goldman SandipVasavada. Radiation exposure to the patient and physician during Sacral Neuromodulation. Journal of urology 2012. http://dx.doi.org/10.1016/j.juro. 2012.02.1737 